\title{
HIV pre-exposure prophylaxis (PrEP)- knowledge and attitudes among a New York City emergency department patient population
}

\author{
Yvette Calderon $^{1 *}$, Jason Leider ${ }^{1}$, Ethan Cowan ${ }^{1}$, Christopher Brusalis², Joanne Mantell ${ }^{3}$, Theo Sandfort ${ }^{1}$ \\ From 17th International Symposium on HIV and Emerging Infectious Diseases (ISHEID) \\ Marseille, France. 23-25 May 2012
}

\section{Background}

HIV Pre-exposure Prophylaxis (PrEP), in which HIV-negative individuals receive antiretroviral medications to prevent HIV acquisition, has shown potential as a means to reduce HIV incidence among high-risk persons. The acceptability of PrEP among at-risk persons will strongly impact the effectiveness of PrEP. This study aimed to assess knowledge and attitudes towards PrEP within a demographically-mixed community with high HIV prevalence.

\section{Materials and methods}

A cross-sectional study was conducted with a convenience sample of Emergency Department (ED) patients at two New York City municipal hospitals. Eligible participants completed an anonymous written survey about knowledge and acceptability of PrEP. Means and standard deviations were calculated for continuous variables and proportions for categorical variables. Standard bivariate methods were used to compare acceptability and knowledge by race, ethnicity and gender.

\section{Results}

474 ED patients agreed to participate. The study population was $40.9 \%$ male, $40.7 \%$ Latino and $38.2 \%$ non-Hispanic Black. 7 participants (1.5\%) self-identified as MSM. Mean age was 35.3, SD +/- 13.1 years. $66.4 \%$ reported inconsistent condom use and $78.4 \%$ had previously had an HIV test. 13.3\% reported knowledge of either the term "PrEP" or the use of antiretroviral medications to prevent HIV acquisition. More people indicated they were unlikely or extremely unlikely to use PrEP (40.1\%) than indicated they would likely take PrEP if available (32.2\%). Many (27.7\%) were unsure if they would or would not take PrEP. $44.4 \%$ thought that individuals would stop using condoms if on PrEP, while $27.0 \%$ thought that individuals would continue using them. Some participants (28.4\%) incorrectly thought that PrEP needed to be taken only prior to sex. There were no differences in knowledge or acceptability of PrEP by gender. Latinos were more likely (17.6\%) than blacks $(8.8 \%)$ and others (12.4\%) to report knowledge of PrEP.

\section{Conclusions}

Potential providers of PrEP must consider limitations in acceptability to this HIV prevention strategy. Future administration of PrEP must incorporate patient education to ensure user understanding of the technology and its potential limitations.

\section{Author details}

${ }^{1}$ Albert Einstein College of Medicine, Bronx, New York, USA. ${ }^{2}$ Jacobi Medical Center, Bronx, New York, USA. ${ }^{3}$ Columbia University, New York, New York, USA.

Published: 25 May 2012

\section{doi:10.1186/1742-4690-9-S1-P94}

Cite this article as: Calderon et al:: HIV pre-exposure prophylaxis (PrEP)knowledge and attitudes among a New York City emergency department patient population. Retrovirology 2012 9(Suppl 1):P94.

*Correspondence: ycal333@yahoo.com

${ }^{1}$ Albert Einstein College of Medicine, Bronx, New York, USA

Full list of author information is available at the end of the article 